\title{
Sensorless Unbalance Correction as an Ancillary Service for LV 4-Wire/3-Phase Power Converters
}

\author{
Andres Suárez-González, Pablo García, Ángel Navarro-Rodríguez, Geber Villa and Jose M. Cano \\ University of Oviedo. Dept.of Elec., Computer \& System Engineering \\ Gijón, 33204, Spain \\ e-mail: suarezandres@uniovi.es, garciafpablo@uniovi.es,navarroangel@uniovi.es, villageber.uo@uniovi.es, jmcano@uniovi.es
}

\begin{abstract}
This paper describes a method to provide LV four-wire three-phase power converters with the capability of correcting unbalance as an ancillary service to the main role that they play in the distribution system (distributed generator, energy storage system, drive, etc). Typically, dedicated grid/load current sensors are needed to effectively comply with unbalance compensation tasks, increasing system cost and reducing reliability. This is due to the difficulties that arises in the extraction of the zero and negative voltage components from the voltages at the PCC, such as the inadequate resolution of full-scaled voltage sensors and limited spectral separation. In this paper, the proposed compensation method does not rely on any more sensors that those typically used in VSCs, and in any case, those sensors are limited to the point of connection of the power converter. High frequency signal injection is evaluated for the compensation. For the zero sequence, an especial arrangement of the converter voltage sensors together with a repetitive controller is used.
\end{abstract}

\section{INTRODUCTION}

The adoption of distributed generation has increased in the last years due to both politics and economics regulations [1], [2], emerging power electronics converters topologies which allow to improve network quality and reliability [3] and decrease in the costs for distributed generation. Power converters have been employed at the transmission network to improve power transmission capability, such as Static Synchronous Compensator (STATCOM) [4], Static Synchronous Series Compensator (SSSC) [5] and Unified Power Flow Controller (UPFC) [4]. Similar topologies have been later integrated at distribution levels i.e. Active Power Filters (APF) [6]-[8], allowing the increase of the distributed generation penetration and overcoming some problems related to the decentralized generation approach.

Among the problems related to distributed generation paradigm [9], [10], current and/or voltage unbalance or sag has recently received much attention [11]-[13]. There are several international standards which recommend maximum limits of the voltage unbalance: ANSI Standard C81.1 requires to keep

The present work has been partially supported by the predoctoral grants program Severo Ochoa for the formation in research and university teaching of Principado de Asturias PCTI-FICYT under the grant ID BP14-135. This work is also supported in part by the Research, Technological Development and Innovation Program Oriented to the Society Challenges of the Spanish Ministry of Economy and Competitiveness under grants ENE2013-44245-R and ENE2016-77919-R and by the European Union through ERFD Structural Funds (FEDER). it within 3\% [14], International Electrotechnical Commission (IEC) and the Comite Europeen de Normalisation Electrotechnique (CENELEC) recommends the limit of 2\% [15], [16].

Unbalance compensation can be achieved by shunt, series, or series/shunt combination of the power converter [17]. Among the three structures, series/shunt allows for an optimal [18] sharing of the voltage/current compensation. However, it requires a dedicated converter for the compensation problem. Solely shunt converter compensation can lead to high compensation currents through the converters, reducing the active/reactive power supply capability, and thus some sharing mechanism among the APF is needed [12]. Sharing of unbalance can be done either by exchanging information among the microgrid converters or by an autonomous method which is implemented in the form of a droop control [12], [13].

Measurement of the unbalanced components, negative and zero sequence voltages and currents, is a challenging task. By one side, use of the negative and zero sequence voltages at the PCC is affected by the ration between the grid impedance and the load to be compensated. By the other, use of negative and zero sequence currents needs for additional current sensors to be placed either at the load or at the grid side. At this paper, it is proposed to overcome these limitations by a combination of novel techniques. Negative sequence will be measured from the converter current sensors and by adding an additional excitation high-frequency signal over the voltage command. This high-frequency signal will interact with the unbalance load, creating a high-frequency negative current that will be used for the compensation. Zero sequence voltage will be measured by using a dedicated voltage sensor, fully scaled to this signal. This will allow to increase the resolution while, in conjunction with another two phase-neutral sensors, will allow the synchronization of the power converter to operate normally. Zero sequence compensation will also explore the use of the high-frequency signal in order to boost the measurement process.

This paper is organized as follows. In section II, a novel modeling technique will be used to analytical highlight the coupling between the positive, negative and zero current sequences with non-balanced loads and voltage unbalances. The proposed analysis allows for obtaining the expressions of the complex current vectors at the $\alpha \beta 0$ reference frame 


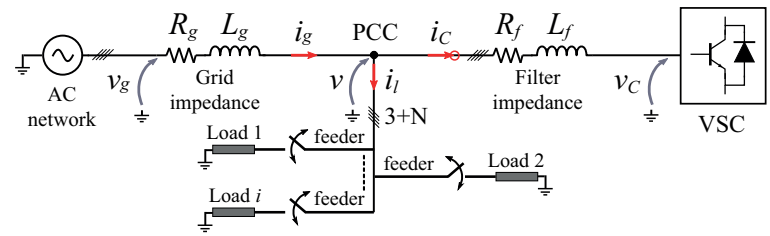

Fig. 1. Schematic for power converter and load connection to the point of common coupling. Loads can be both $3 \phi$ or $1 \phi$.

from the values of the voltages and loads at the $a b c$ reference frame. The method is valid both for the analysis at the fundamental frequency, but also when a high frequency signal is injected by the converter. In section III, the proposed compensation method for the negative sequence current using a high frequency signal injection for the measurement of the unbalance from the converter-side current sensors is explained. The development includes the needed theoretical expressions as well as the signal processing. In section IV, the proposed zero sequence compensation is included. The method includes to main features: 1) the use of an special sensor arrangement in order to increase the resolution of the zero sequence voltage and 2) the use of a repetitive controller for the compensation of the zero sequence current. Finally, in section V, simulation results both for the negative sequence current and the zero sequence voltage are shown.

\section{SYSTEM MODELING}

Modelling of the system shown in Fig. 1 can be done both in the stationary reference frame $(\alpha, \beta, 0)$ or in a synchronous reference frame $(d, q, 0)$. In this paper, the $\alpha \beta 0$ reference frame is used, since it leads to a simpler formulation in the presence of unbalances [19]. Considering $R L$ elements for describing the system impedances, the voltage equation (which can be applied between any two nodes, and particularly at the PCC, in Fig. 1) is given by (1).

$$
\mathbf{v}_{a b c}=\mathbf{R}_{a b c} \mathbf{i}_{a b c}+\mathbf{L}_{a b c} \frac{d \mathbf{i}_{a b c}}{d t}
$$

Transformation from $a b c$ to $\alpha \beta 0$ is defined in (2a) for the currents and voltages and in (2b) for the impedances, being the A matrix transformation defined in (3)

$$
\begin{gathered}
\mathbf{x}_{a b c}=\mathbf{A} \mathbf{x}_{\alpha \beta 0} \\
\mathbf{Z}_{\alpha \beta 0}=\mathbf{A}^{-1} \mathbf{Z}_{a b c} \mathbf{A} \\
\mathbf{A}=\sqrt{\frac{2}{3}}\left(\begin{array}{ccc}
1 & 0 & \frac{1}{\sqrt{2}} \\
-\frac{1}{2} & \frac{\sqrt{3}}{2} & \frac{1}{\sqrt{2}} \\
-\frac{1}{2} & -\frac{\sqrt{3}}{2} & \frac{1}{\sqrt{2}}
\end{array}\right)
\end{gathered}
$$

Unbalances in the distribution network impedance can be represented in the $\alpha \beta 0$ reference frame using the following procedure. The general model for the impedance matrix in an stationary reference frame aligned with the spatial angle of the load $i$ ( $\theta_{e}^{i}$ in Fig. 2), and neglecting the cross-coupling between the phases, is represented by equation (4)

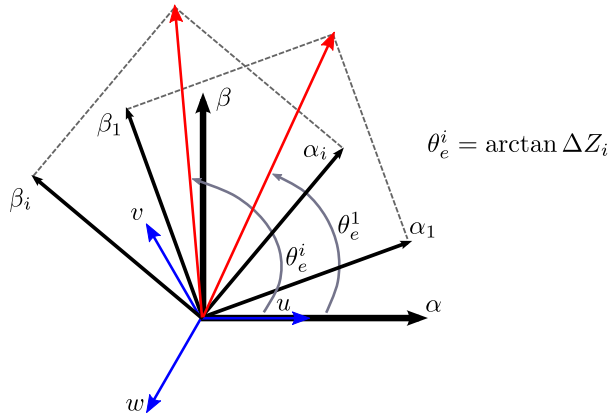

Fig. 2. Axis orientation for system loads $\left(\alpha_{i} \beta_{i}\right)$ and general stationary reference frame axis $(\alpha \beta)$.

$$
\mathbf{Z}_{\alpha \beta \mathbf{0}_{i}}=\left(\begin{array}{ccc}
Z_{\alpha \alpha i} & 0 & 0 \\
0 & Z_{\beta \beta_{i}} & 0 \\
0 & 0 & Z_{00 i}
\end{array}\right)
$$

on (4), each load is represented by the $i$ subscript. For the case the load is balanced, $Z_{\alpha \alpha i}=Z_{\beta \beta_{i}}=Z_{00 i}$. Rotating the impedance matrix to the $\alpha \beta 0$ reference frame and considering $n$ loads, leads to (5).

$$
\begin{aligned}
\mathbf{Z}_{\alpha \beta 0} & =\sum_{i=1}^{n}\left[\Sigma Z_{i}\left(\begin{array}{ccc}
1 & 0 & 0 \\
0 & 1 & 0 \\
0 & 0 & 1
\end{array}\right)\right. \\
& \left.+\Delta Z_{i}\left(\begin{array}{ccc}
\cos \theta_{e}^{i} & -\sin \theta_{e}^{i} & \sqrt{2} \cos \theta_{e}^{i} \\
-\sin \theta_{e}^{i} & -\cos \theta_{e}^{i} & \sqrt{2} \sin \theta_{e}^{i} \\
\sqrt{2} \cos \theta_{e}^{i} & \sqrt{2} \sin \theta_{e}^{i} & 0
\end{array}\right)\right]
\end{aligned}
$$

In (5), $\Sigma Z_{i}$ and $\Delta Z_{i}$ can be defined as (6) and (7) respectively.

$$
\begin{gathered}
\Sigma Z_{i}=\frac{Z_{\alpha \alpha i}+Z_{\beta \beta_{i}}}{2}=\frac{Z_{a}+Z_{b}+Z_{c}}{3} \\
\Delta Z_{i}=\frac{Z_{\alpha \alpha i}-Z_{\beta \beta_{i}}}{2}=\frac{Z_{a}+a \cdot Z_{b}+a^{2} \cdot Z_{c}}{3}
\end{gathered}
$$

$\theta_{e}^{i}$ is the spatial angular phase of the unbalance load and $a=$ $e^{j 2 \pi / 3}$. For example, for single phase loads at phases $u, v, w$, $\theta_{e}^{i}$ equals $0,2 \pi / 3$ and $4 \pi / 3$ respectively.

By substituting (5) in the voltage equation (1) expressed in the $\alpha \beta 0$ reference frame, and considering steady state conditions, the resulting current vector $\left(\mathbf{i}_{\alpha \beta}\right)$ is given by (8) and the homopolar component $\left(i_{0}\right)$ by $(9)$.

$$
\begin{array}{r}
\mathbf{i}_{\alpha \beta}=V_{\alpha \beta}^{+} \sum_{i=1}^{n}\left[\Sigma Y_{i} e^{j \omega_{e} t}+\Delta Y_{i} e^{-j\left(\omega_{e} t+\theta_{e}^{i}\right)}\right] \\
+V_{\alpha \beta}^{-} \sum_{i=1}^{n}\left[\Sigma Y_{i} e^{-j \omega_{e} t}+\Delta Y_{i} e^{j\left(\omega_{e} t-\theta_{e}^{i}\right)}\right] \\
i_{0}=\sum_{i=1}^{n}\left[V_{\alpha \beta}^{0} \frac{1}{\Sigma Z_{i}} \sin \left(\omega_{e} t+\phi_{e}\right)\right. \\
\left.+\frac{\Delta Y_{i}}{\Sigma Y_{i}} \sqrt{2} I_{\alpha \beta} \sin \left(\omega_{e} t+\theta_{e}^{i}\right)\right]
\end{array}
$$

where $\Sigma Y_{i}=\frac{\Sigma Z_{i}}{\Sigma Z_{i}{ }^{2}-\Delta Z_{i}{ }^{2}}, \Delta Y_{i}=-\frac{\Delta Z_{i}}{\Sigma Z_{i}{ }^{2}-\Delta Z_{i}^{2}}, \omega_{e}$ is the grid frequency, $\phi_{e}=\arctan \left(\mathbf{v}_{\alpha \beta}\right), I_{\alpha \beta}$ is the magnitude of 
the current vector and $V_{\alpha \beta}^{+}, V_{\alpha \beta}^{-}$and $V_{\alpha \beta}^{0}$ are the positive, negative and zero sequence magnitudes of the voltage vector.

The above expressions have been evaluated by numerical simulation. For that, different changes at the phase impedance values as well as the phase voltages were applied. The results given by the proposed expressions have been compared with the results of a simulation in the $a b c$ reference frame and validated once both approaches were in close agreement.

Figures 3, 4 and 5 show, respectively, the voltage vector at the $\alpha \beta 0$ reference frame, the considered grid impedance and current vector at the $\alpha \beta 0$ reference frame. The simulation is carried out for a three phase system in a wye-connection with neutral configuration. The different test conditions are following enumerated: Initially, the system is at balanced condition. As expected, $\Delta Z=0$ and the angle of the asymmetry is also hold at zero. At $t=0.1 \mathrm{~s}$, the impedance from the three phases is changed from $180 \mathrm{deg}$ to $60 \mathrm{deg}$ for phase $a$, from $180 \mathrm{deg}$ to $-90 \mathrm{deg}$ for phase $b$ and from $180 \mathrm{deg}$ to $90 \mathrm{deg}$ for phase $c$. Consequently both $\Sigma Z$ and $\Delta Z$ vary from $1 p . u$. to 0.33 p.u and from 0 p.u. to 0.7 p.u. respectively. It is also important to note that the $\theta$ value is equal to the unbalance orientation, resulting in a value of $21 \mathrm{deg}$. In summary, the unbalance level is codified by the $\Delta Z$ level and the $\theta$ angle provides its direction. The effect of the unbalance is coupled to the current vector components, as shown in 5 . When the impedance unbalance is applied, the negative sequence component of the current vector $\left(I_{\alpha \beta}^{-}\right)$is modulated according to the developed expressions. Considering the neutral connection, an homopolar current $\left(I_{0}\right)$ flows into the system.

Effects of a direct unbalance of the voltage vector at the PCC is analyzed starting at $t=0.2 \mathrm{~s}$. Phase $b$ is lagged 90 respect to previous situation. Simultaneously, the impedance of phase $b$ and $c$ is changed to zero and the module of phase $c$ is decreased to 0.7 p.u. As expected, this causes a negative sequence voltage to be applied into the system and a variation in the resulting zero sequence voltage. Finally, at $t=0.3 \mathrm{~s}$, the voltage disturbance is cleared but the impedance values are kept unbalanced with $\left|Z_{a}\right|=0.8$ p.u., $\left|Z_{b}\right|=1$ p.u. and $\left|Z_{c}\right|=0.7$ p.u. This results in a variation of the negative sequence current and in the homopolar voltage as well.

From (8), it is clear load unbalances will cause negative and zero sequence currents to appear, which potentially will create an unbalance in the grid supplied voltages. Compensation can be done by injecting a negative and zero sequence currents in opposition to the respective unbalanced currents [12]. There are several methods proposed in the literature to compensate for this, all of them requires to separate (filter) the negative and zero sequences from the positive one and to cancel it out by using negative and zero sequence current controllers [12], [13]. In the following section, a compensation method based on the injection of a high frequency signal is proposed. The method will enable the measurement of the grid impedance from the converter side currents, thus leading to a sensorless compensation.
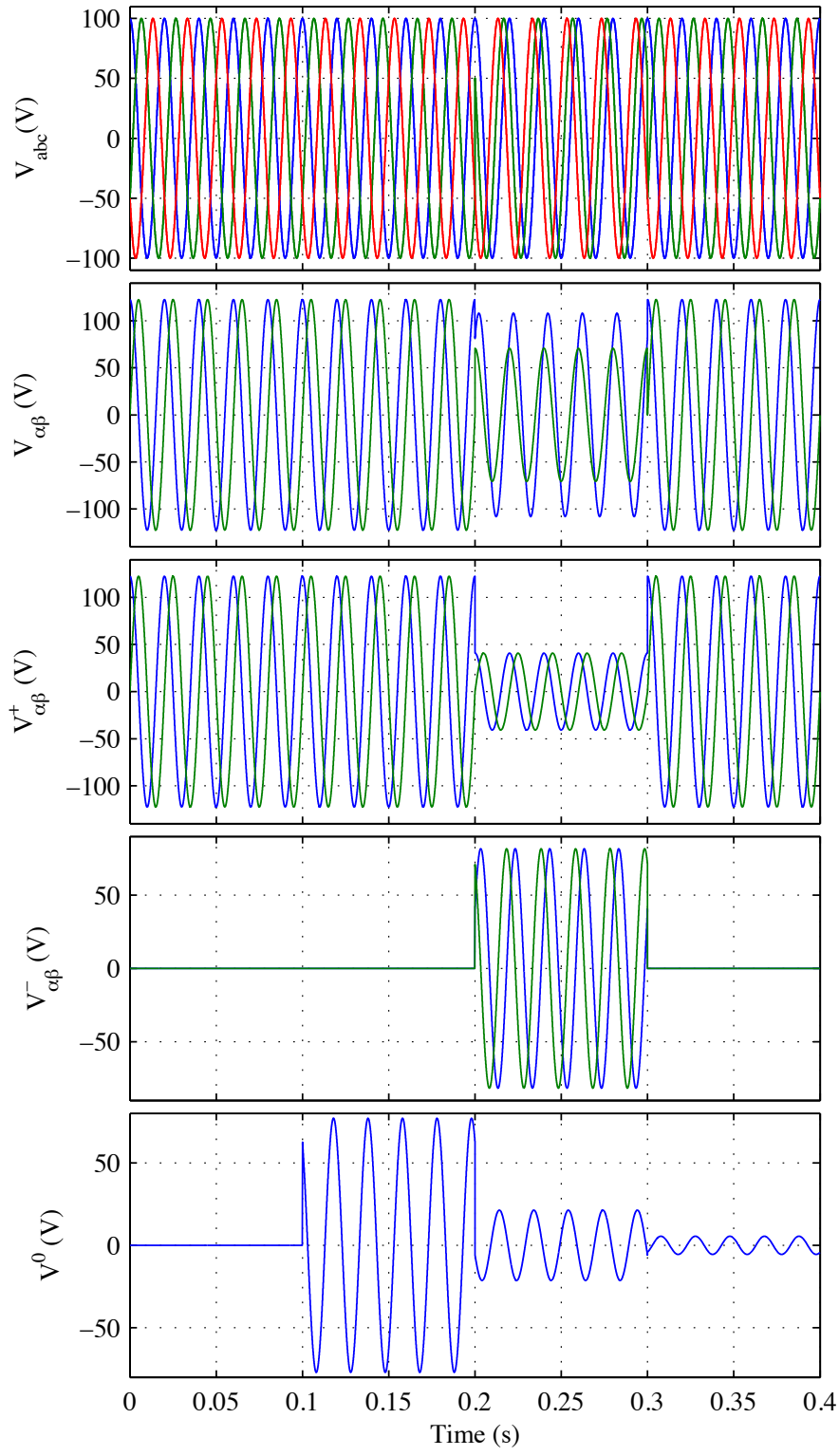

Fig. 3. Simulation results for the voltage at the PCC. From top to bottom: a) phase voltages, b) $\alpha, \beta$ components, c), d) and e) are the positive, negative and zero components of the voltage vector.

\section{HFS-BASED UNBALANCE COMPENSATION}

The current equation (8) is valid for any excitation voltage with fixed frequency. Adding a balanced high frequency $\left(\omega_{c}\right)$ carrier-signal voltage, $\mathbf{v}_{\alpha \beta}^{h f+}$, as denoted by (10), to the voltage at the PCC, results in a high frequency current (11) that shows a negative sequence component which is determined by the impedance unbalance. However, two fundamental differences exist compared to the fundamental excitation: 1) as the high frequency voltage is created by the converter and superimposed to the fundamental voltage, the resulting high frequency converter current, $\mathbf{i}_{\mathbf{C}}^{h f}$, contains the unbalance information, hence, is not needed to measure the current at the load, $\mathbf{i}_{\mathbf{l}}$, or at the grid side, $\mathbf{i}_{\mathbf{g}}$, to proceed with the compensation (see 

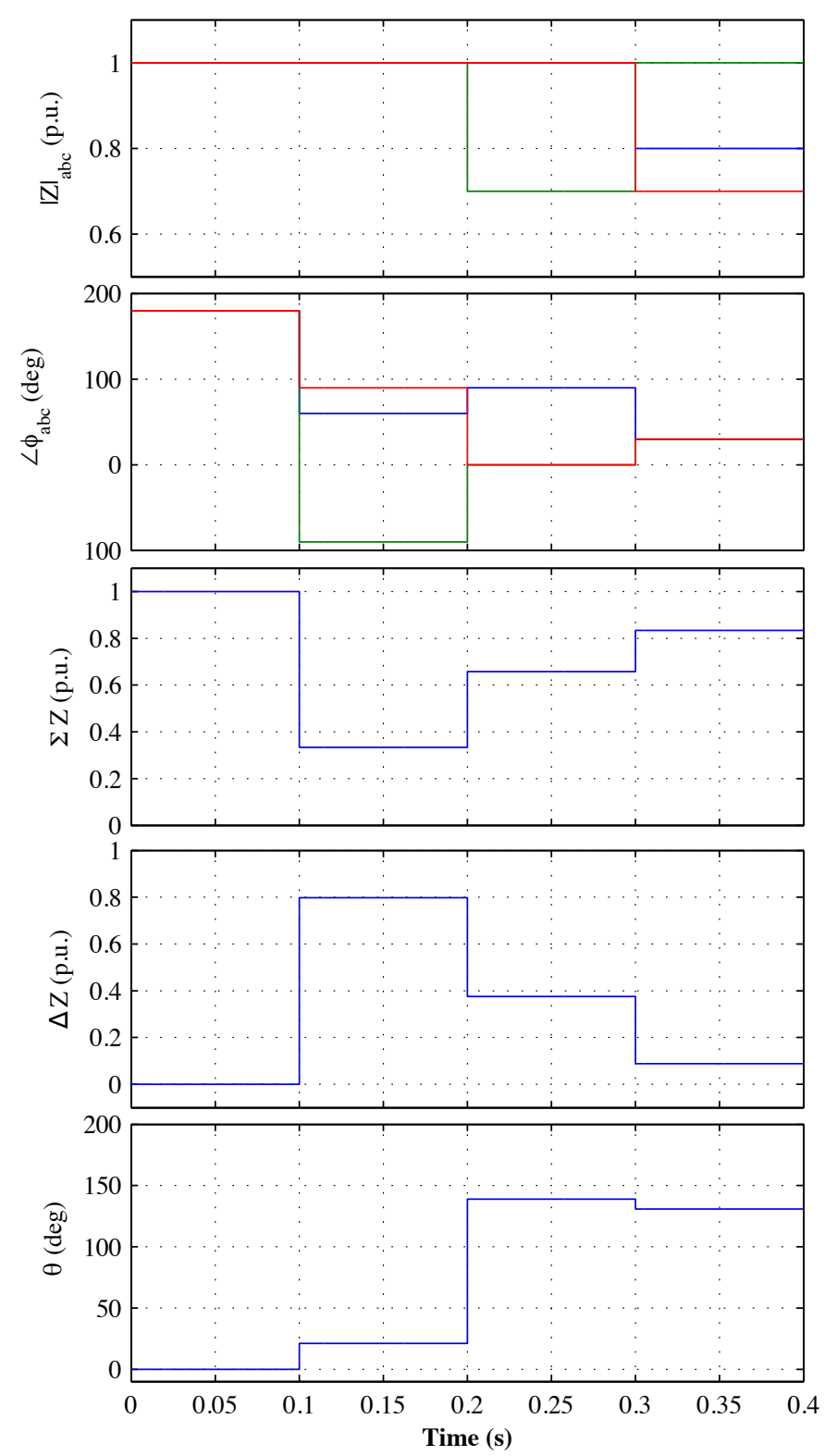

Fig. 4. Simulation results for the grid impedance as seen from the PCC. From top to bottom: a) module of the impedance, b) phase of the impedance, c) mean impedance, d) differential impedance and e) asymmetry angle.

Fig. 1); 2) filtering the negative sequence current from the positive one is easier at higher frequencies, since there is more spectral separation. This will allow to increase the resolution of the system to light unbalanced conditions.

$$
\begin{gathered}
\mathbf{v}_{\alpha \beta}^{h f}=V^{h f} e^{j \omega_{c} t} \\
\mathbf{i}_{\alpha \beta}^{h f}=V^{h f} \sum_{i=1}^{n}\left[\Sigma Y_{i}^{h f} e^{j \omega_{c} t}+\Delta Y_{i}^{h f} e^{j\left(-\omega_{c} t+\theta_{e}^{i}\right)}\right]
\end{gathered}
$$

Adding the appropriate value of negative sequence high frequency voltage allows to fully compensate the negative
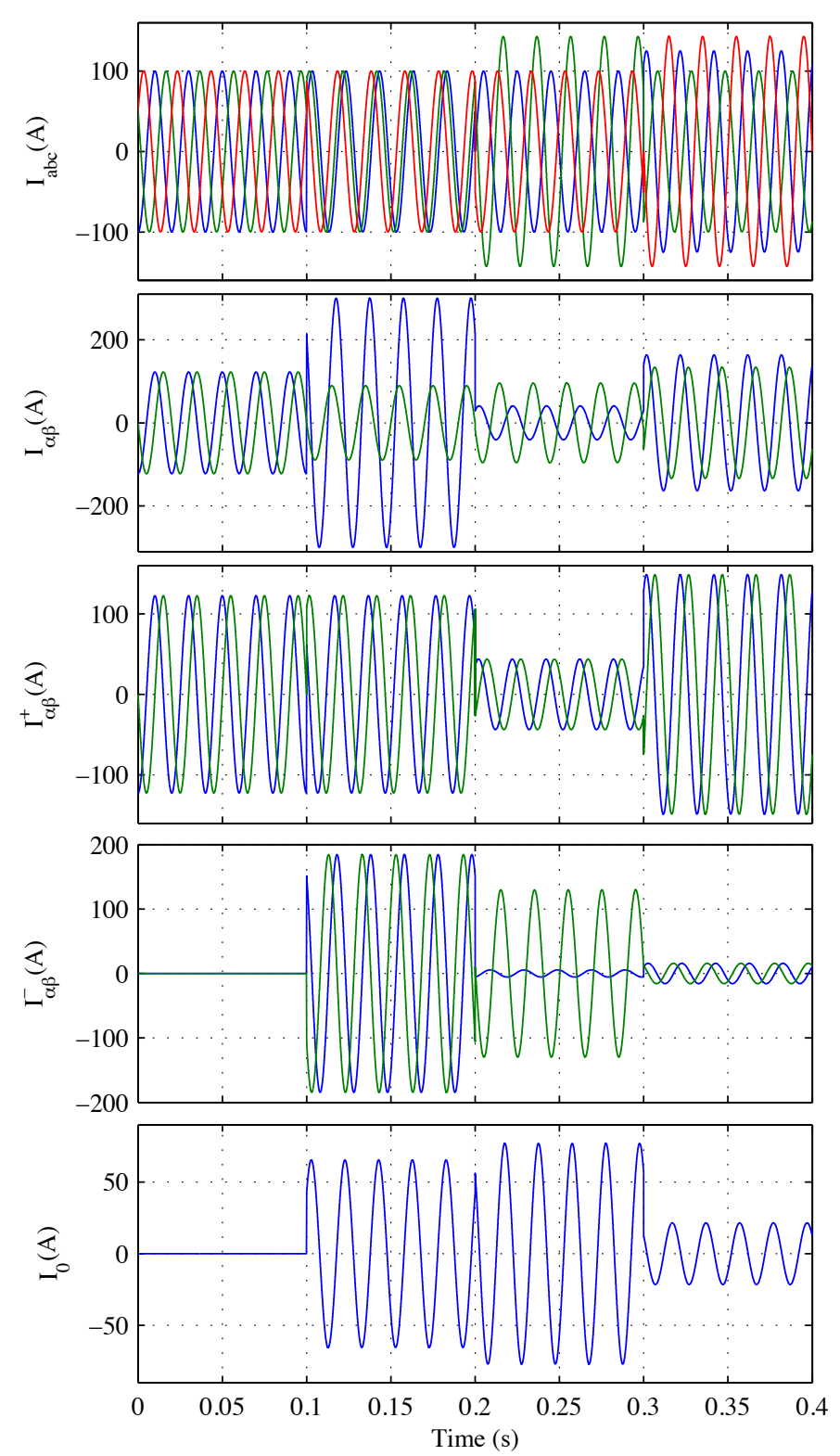

Fig. 5. Simulation results for the grid currents at the PCC. From top to bottom: a) phase currents, b) $\alpha, \beta$ components, c), d) and e) are the positive, negative and zero components of the current vector.

sequence current arising from (11). Applying (8) to the carrier frequency and forcing $\mathbf{i}_{\mathbf{C} \alpha \beta}^{h f-}$ to be zero leads to

$$
\mathbf{v}_{\alpha \beta}^{h f-}=\mathbf{v}_{\alpha \beta}^{h f+} \frac{-\Delta Y_{i}^{h f} e^{j \theta_{e}^{i}}}{\Sigma Y_{i}^{h f}} .
$$

As the information of the load unbalance is still included in $\mathbf{v}_{\alpha \beta}^{h f-}$, the proper correction action at grid frequency can be derived from this value.

In regard to the high frequency excitation signal, its magnitude and frequency values can be tuned separately. From a power quality perspective, increasing the frequency and reducing the magnitude is preferred. However, this reduces the signal to noise ratio of the method. Additionally, the injected 


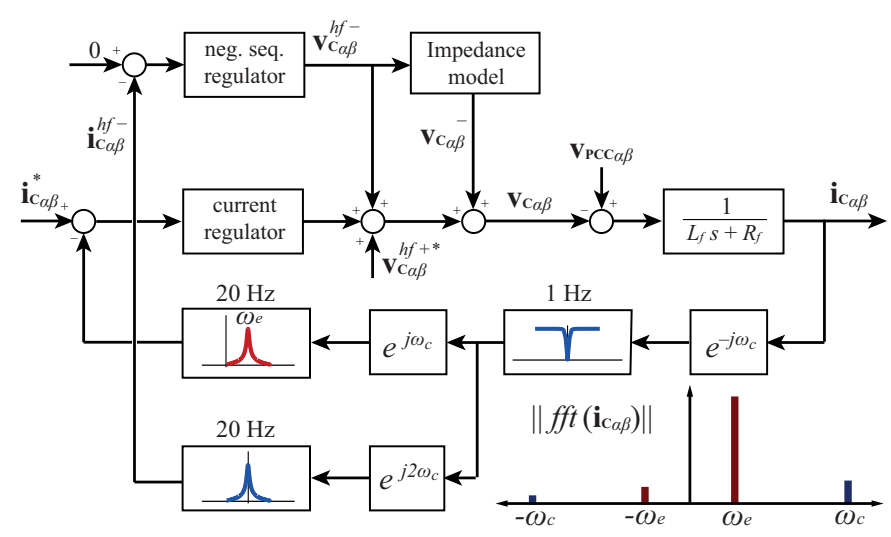

Fig. 6. High frequency signal injection and signal processing. Spectrum showing the main components of the converter current $\left(\mathbf{i}_{\mathbf{c}}\right)$ is shown on the lower right corner.

frequency should not be a harmonic of the fundamental frequency, in order to avoid the reaction of any existing harmonic compensator connected to the grid [20]. For this paper, the values shown at Table I have been chosen.

The control scheme shown in Fig. 6 is used to compensate for the negative sequence current at grid frequency. A positive sequence high frequency $(333 H z)$ carrier voltage, $\mathbf{v}_{\mathbf{C} \alpha \beta}^{h f+}$, is added to the converter voltage, causing a negative sequence component to appear in the converter current at the same frequency, $\mathbf{i}_{\mathbf{C} \alpha \beta}^{h f-}$. Provided that the grid side is free from this frequency component and mostly balanced, this negative sequence component is the same that could be observed at the load, i.e. $\mathbf{i}_{\mathbf{C} \alpha \beta}^{h f-}=-\mathbf{i}_{\mathbf{l} \alpha \beta}^{h f-}$. This negative sequence current is obtained from the converter current and controlled to be zero by using two PI regulators, one per each axis. The output of this regulator, $\mathbf{v}_{\mathbf{C} \alpha \beta}^{h f-}$, is used to estimate the negative sequence to be added at grid frequency to the converter voltage, $\mathbf{v}_{\mathbf{C} \alpha \beta}^{-}$, in order to achieve the compensating objective. An unbalance impedance model is used to relate injections at both frequencies, provided that a linear load is connected to the PCC.

\section{ZERO-SEQUENCE COMPENSATION}

Zero-sequence currents arises in the LV distribution system as a consequence of both unbalanced and non-linear loads. The local compensation of zero-sequence currents in order to avoid their propagation upstream from the PCC is a desirable ancillary service for power converters, though only four-wire topologies are valid to comply with this function. This task can be easily done by measuring the zero-sequence current at the grid side and implementing a closed loop strategy for compensating it. Unfortunately, this requires not only an additional sensor but also the connection or communication between distant points. A theoretical alternative comes from the compensation of the zero-sequence voltage at the PCC, which could be calculated from the grid phase-voltage sensors of the VSC. However, the use of three voltage sensors ranged

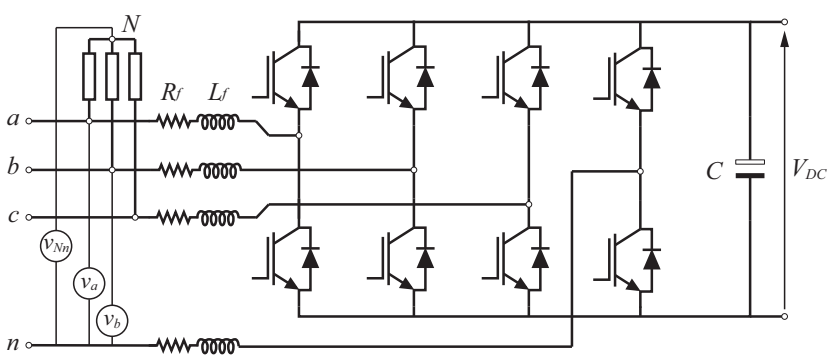

Fig. 7. Zero sequence voltage compensation. Proposed sensor arrangement for the measurement of the zero sequence component.

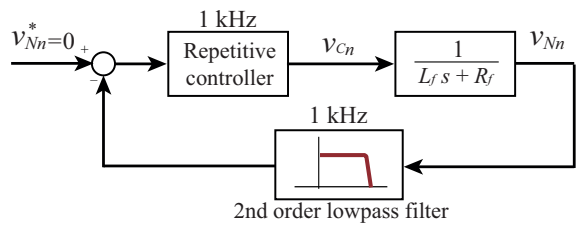

Fig. 8. Zero sequence voltage compensation. Control loop modeling based on the use of a repetitive controller.

at full scale to measure the voltages at the PCC makes it difficult to provide a suitable resolution in the calculation of this value, and can be even impossible in the interconnection to strong power systems. The arrangement proposed in Fig. 7 for a four-leg topology is used in this paper to solve this adversity. Two full-scale sensors are combined with a third one, ranged at a much lower scale. The latter sensor is devoted to measure the zero-sequence voltage component directly, by connecting it between the grounded neutral point of the installation and the artificial neutral point of a three-phase balanced high impedance load, intentionally located at the converter POC. The analytical expression for the zero sequence voltage is given by (13).

$$
v_{N n}=\frac{1}{3}\left(v_{a}+v_{b}+v_{c}\right)=v^{0} .
$$

After applying a second-order low-pass filter to remove the high frequency components of $v_{N_{n}}$, a simple resonant controller could be used to cancel the fundamental frequency zerosequence component seen from the grid side. Nevertheless, a repetitive controller (RC) was selected for this application in order to also remove other zero-sequence frequencies. This is of especial interest in this type of installations in which the massive use of single-phase non-linear loads can cause a significant flow of tripplen current harmonics. Fig. 8 shows the control loop, which cancels the zero-sequence components by adding the proper signal to the modulation of the neutral leg of the converter. Notice that, although the total modulation of this leg contains other zero-sequence components to counteract the effect of the traditional zero sequence injection in the other legs, only the added output of the RC appears in $v_{C n}$.

\section{Simulation RESUlTS}

For the simulation results, the network topology shown in Fig. 1 is used. The loads connected to the PCC are a 
TABLE I

SYSTEM PARAMETERS

\begin{tabular}{c|c}
\hline Converter parameters & Value \\
\hline Power [kW] & 100 \\
DC Link Voltage [V] & 850 \\
Max Current [A] & 200 \\
Switching frequency [Hz] & 5000 \\
AC Filter & $L_{f}=5 m H, R_{f}=0.2 \Omega$ \\
Grid impedance & $L_{g}=1 m H, R_{g}=0.02 \Omega$ \\
DC bus capacitor $[\mu F]$ & 4400 \\
\hline Loads & \\
\hline Three phase balance load & $R_{l}=18 \Omega$ \\
Single phase loads & $R_{l s}=18 \Omega$ \\
\hline Control tuning & \\
\hline$T_{c}[\mu s]$ & 100 \\
DC voltage loop & $K_{p}=0.2212, K_{i}=80$ \\
Current control loop & $K_{p}=55.2920, K_{i}=15$ \\
\hline High frequency parameters & \\
\hline Carrier voltage [V] & 10.0 \\
Carrier frequency [Hz] & 333 \\
\hline
\end{tabular}
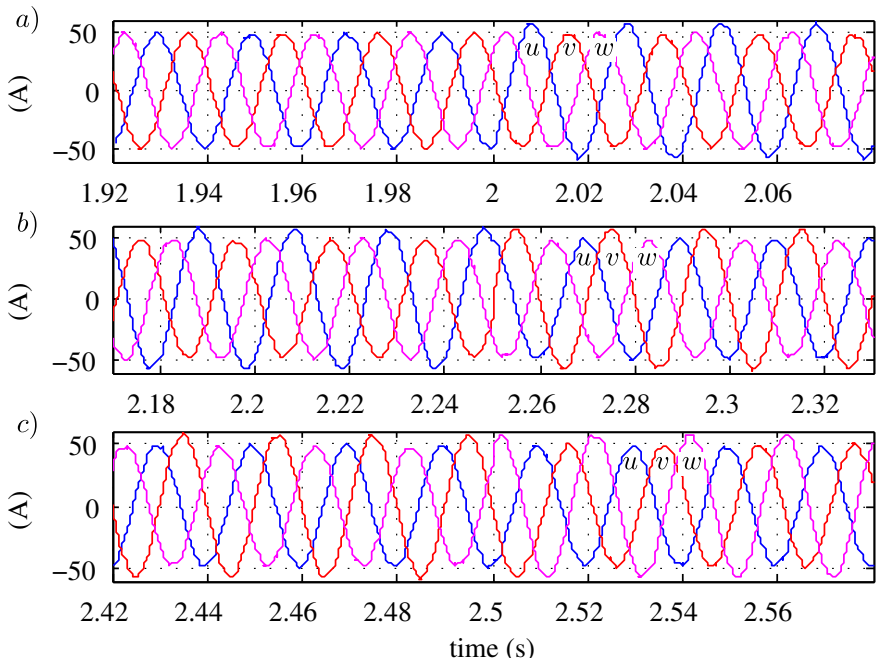

Fig. 9. Simulation results. Phase currents at grid for single phase loads connected at phases $a), u, t=2 s ; b), v(u$ disconnected $), t=2.25 s ; c), w(v$ disconnected), $t=2.5 \mathrm{~s}$. Load is resistive for the three cases $R_{l}=13.25 \Omega$.

three phase $R L$ load and three single phase $R$ loads between phases $u, v, w$ and the neutral. These single phase loads are sequentially connected and disconnected in order to see the effects on the negative sequence currents. The parameters for the simulation are given at Table I. Phase grid currents when the single phase loads are connected are shown at Fig 9. As clearly shown, the unbalance at the grid current is noticeable, being, as expected, the phase carrying more current the one at which the load is connected. The magnitudes of the negative sequence currents for the grid and converter current vectors are represented at Fig. 10 a), b) respectively. From Fig. 10 it is clear that both currents have information about the unbalance. However, using the converter current has important drawbacks due to the poor transient response. On the other side, using the grid current needs for additional current sensors in the grid side or at each feeder, which increases the system costs and decreases the reliability. For improving the unbalance measurement using only the converter side current sensors, a

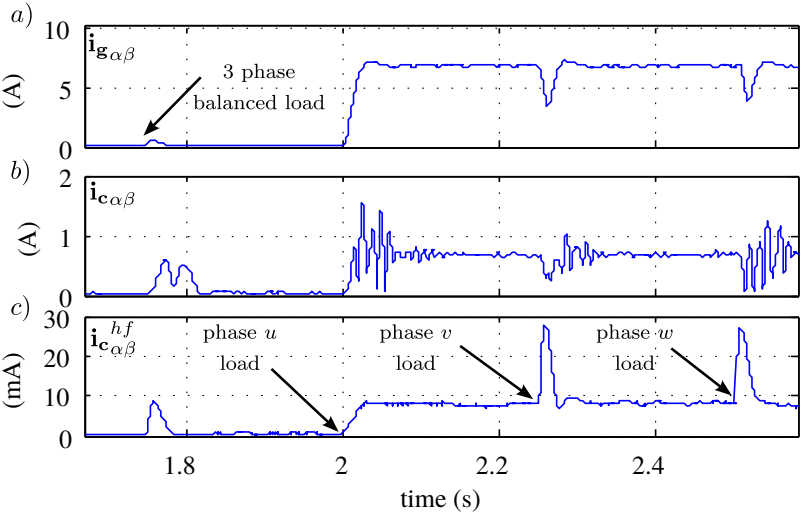

Fig. 10. Simulation Results. Negative sequence component magnitude resulting from single phase load connection at the PCC. $a$ ), negative sequence current $(-50 \mathrm{~Hz})$ for grid current vector; $b)$, negative sequence current $(-50 \mathrm{~Hz})$ for converter current vector; $c)$ negative sequence carrier current $(-333 \mathrm{~Hz})$ for converter current vector.

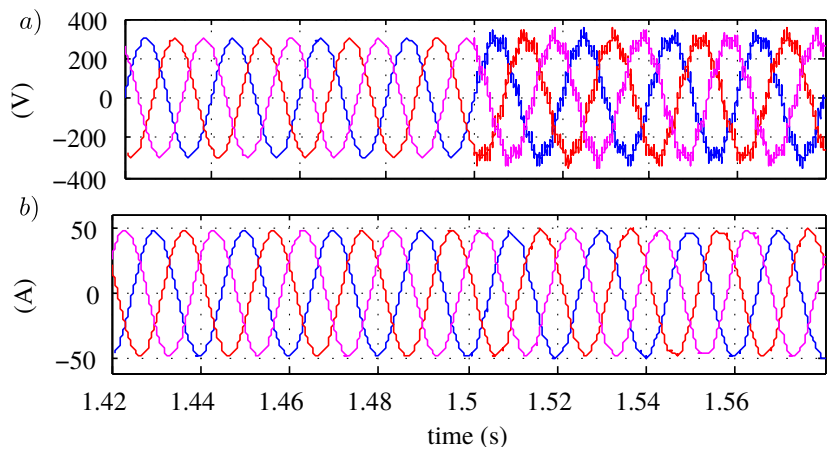

Fig. 11. Simulation results. $a$ ) phase voltages at PCC, $b$ ) phase currents at grid. High frequency signal injection is activated at $t=1.5 \mathrm{~s}$. THD is $6 \%$ and $3 \%$ for the voltages and the currents respectively.

high frequency signal is injected by the converter, as shown in Fig. 6. The high frequency signal added to the fundamental voltage command is shown at Fig. 11. Obviously, the injected high frequency current increases the resulting THD, both in the currents and in the voltages at the PCC. However, for the parameters shown at Table I, the THD is below the maximum allowed by international standards [14]-[16]. The resulting high frequency negative sequence current (being for the chosen high frequency frequency at $-333 \mathrm{~Hz}$ ) is shown at Fig. $10 \mathrm{c}$ ). From the figure, it is clear that the transient response is far better compared to the fundamental negative sequence current (Fig. $10 \mathrm{~b}$ ). It is worth noting that the same bandwidth is used for isolating the negative sequence and the negative carrier currents (see Fig. 6). The transient shown when the three phase balance load is connected is because the filter used to isolate the negative carrier current can not reject the high frequency components from the transient. Alternatives based on the use of observers, already proposed for sensorless control of $\mathrm{AC}$ drives can be used if needed. However, it is considered that the compensation of the negative sequence current is an steady state phenomena and, even if fast transient response is desired, it is not an strong requirement. 


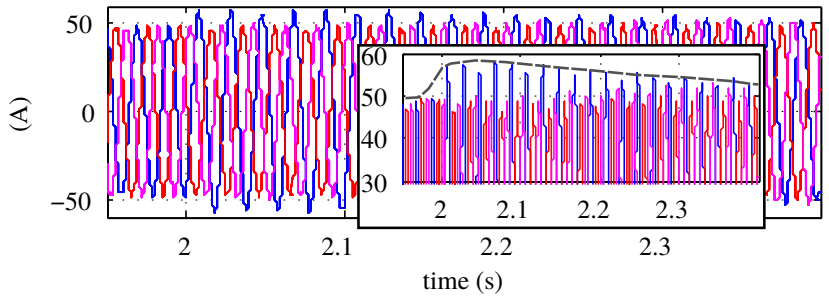

Fig. 12. Simulation results. Grid phase currents. Unbalance at phase $u$ at $t=2 s$. Compensation using the high frequency negative sequence current is activated at $t=2.1 \mathrm{~s}$. Zoomed $y$ axis is represented in the box.
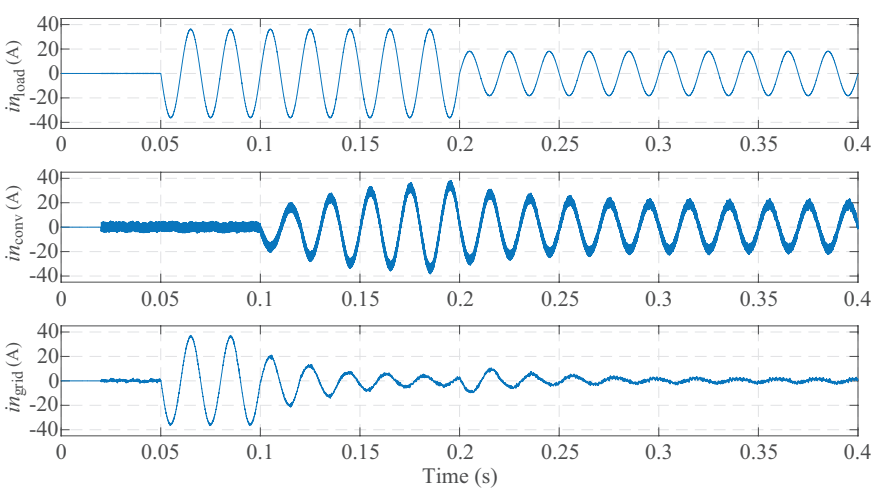

Fig. 13. Simulation results. Zero sequence compensation. (a) neutral current at the load, (b) current at the converter neutral leg, and (c) neutral current at the grid side.

The compensation results for the grid phase currents are shown at Fig 12. The unbalance, though not totally compensated, is noticeable reduced. In Fig. 13, the proper compensation of the zero sequence component is demonstrated. The neutral current at the load is presented in Fig. 13.a). As it can be seen, two single-phase loads are connected to phase $u$ at time $0.05 \mathrm{~s}$ and one of them is disconnected at time $0.2 \mathrm{~s}$ to test transient performance. The application of the RC proposed in this paper together with the special arrangement of voltage sensors, leads to a fast compensation of grid neutral current by the converter.

\section{CONClusions}

In this paper, a method for measuring and compensating unbalance currents for 4-wire 3-phase converters is presented. A high frequency signal injection is used for the negative sequence and a RC for zero sequence components. Both strategies allow a sensorless high-resolution alternative for unbalance compensation. A model considering unbalance loads and voltages has been developed. This model allows to estimate the negative sequence currents generated by the unbalance loads as well as by the cross coupling terms. From the general model, a high frequency model which show the resulting high frequency current in the presence of unbalance conditions has been obtained.

\section{REFERENCES}

[1] R. Lasseter, "Microgrids," in Power Engineering Society Winter Meeting, 2002. IEEE, vol. 1,2002 , pp. $305-308$ vol.1.
[2] R. Lasseter and P. Paigi, "Microgrid: a conceptual solution," in Power Electronics Specialists Conference, 2004. PESC 04. 2004 IEEE 35th Annual, vol. 6, june 2004, pp. 4285 - 4290 Vol.6.

[3] J. M. Guerrero, "Editorial special issue on power electronics for microgrids; part ii," Power Electronics, IEEE Transactions on, vol. 26, no. 3, pp. $659-663$, march 2011.

[4] N. G. Hingorani and L. Gyugyi, Understanding FACTS: Concepts and Technology of Flexible AC Transmission Systems. New York: IEEE, 2000.

[5] L. Gyugyi, C. Schauder, and L. Sen, "Static synchronous series compensator: A solid-state approach to the series compensation of transmission lines," IEEE Transactions on Power Delivery, vol. 12, no. 1, pp. 406417, 1997.

[6] B. Singh, K. Al-Haddad, and A. Chandra, "A review of active filters for power quality improvement," Industrial Electronics, IEEE Transactions on, vol. 46, no. 5, pp. 960 -971, oct 1999.

[7] V. Corasaniti, M. Barbieri, P. Arnera, and M. Valla, "Comparison of active filters topologies in medium voltage distribution power systems," in Power and Energy Society General Meeting - Conversion and Delivery of Electrical Energy in the 21st Century, 2008 IEEE, july 2008, pp. $1-8$.

[8] H. Fujita and H. Akagi, "The unified power quality conditioner: The integration of series and shunt-active filters," IEEE Transactions on Power Electronics, vol. 13, no. 2, pp. 315-322, 1998.

[9] F. Bastiao, P. Cruz, and R. Fiteiro, "Impact of distributed generation on distribution networks," in Electricity Market, 2008. EEM 2008. 5th International Conference on European, may 2008, pp. 1 -6.

[10] R. Walling, R. Saint, R. Dugan, J. Burke, and L. Kojovic, "Summary of distributed resources impact on power delivery systems," Power Delivery, IEEE Transactions on, vol. 23, no. 3, pp. 1636 -1644, july 2008.

[11] V. Khadkikar, "Enhancing electric power quality using upqc: A comprehensive overview," IEEE Transactions on Power Electronics, vol. 27, no. 5, pp. 2284-2297, 2012.

[12] M. Savaghebi, A. Jalilian, J. Vasquez, and J. Guerrero, "Autonomous voltage unbalance compensation in an islanded droop-controlled microgrid," IEEE Transactions on Industrial Electronics, vol. PP, no. 99, p. 1, 2012.

[13] C. Po-Tai, C. Chien-An, L. Tzung-Lin, and K. Shen-Yuan, "A cooperative imbalance compensation method for distributed-generation interface converters," IEEE Transactions on Industry Applications, vol. 45, no. 2, pp. 805-815, 2009.

[14] American National Standard for Electrical Power Systems and Equipment, Voltage Ratings (60 Hertz). ANSI c84.1-1995.

[15] Voltage characteristics of electricity supplied by public distribution systems. EN50160, 2011.

[16] Electromagnetic compatibility (EMC) Part 4-30:Testing and measurement techniques and Power quality measurement methods. IEC 6100-430, 2003.

[17] V. Khadkikar and A. Chandra, "Upqc-s: A novel concept of simultaneous voltage sag/swell and load reactive power compensations utilizing series inverter of upqc," Power Electronics, IEEE Transactions on, vol. 26, no. 9 , pp. $2414-2425$, sept. 2011.

[18] A. Al-Zamil and D. Torrey, "A passive series, active shunt filter for high power applications," Power Electronics, IEEE Transactions on, vol. 16, no. 1, pp. $101-109$, jan 2001.

[19] J. Guerrero, M. Savaghebi, and R. Teodorescu, "Modeling, analysis, and design of stationary reference frame droop controlled parallel threephase voltage source inverters," in Power Electronics and ECCE Asia (ICPE ECCE), 2011 IEEE 8th International Conference on, June 2011, pp. 272-279.

[20] F. Briz, D. Diaz-Reigosa, M. Degner, P. Garcia, and J. Guerrero, "Dynamic behavior of current controllers for selective harmonic compensation in three-phase active power filters," in Energy Conversion Congress and Exposition (ECCE), 2011 IEEE, June 2011, pp. 28922899. 